\title{
A contact investigation after exposure to a child with disseminated tuberculosis mimicking inflammatory bowel disease
}

Dongsub Kim, MD', Sodam Lee, MS², Sang-Hee Kang, BS², Mi-Sun Park, PhD², So-Young Yoo, MD, PhD³, Tae Yeon Jeon, MD, PhD², JoonSik Choi, MD', Bora Kim, BS ${ }^{4}$, Jong Rim Choi, PhD ${ }^{4}$, Sun Young Cho, MD, PhD ${ }^{4,5}$, Doo Ryeon Chung, MD, PhD ${ }^{4,5}$, Yon Ho Choe, MD, PhD', Yae-Jean Kim, MD, PhD'

${ }^{1}$ Department of Pediatrics, Samsung Medical Center, Sungkyunkwan University School of Medicine, Seoul, ${ }^{2}$ Department of TB Epidemic Investigation, Center for Disease Prevention, Korea Centers for Disease Control and Prevention, Cheongju, ${ }^{3}$ Department of Radiology, Samsung Medical Center, Sungkyunkwan University School of Medicine, Seoul, ${ }^{4}$ Center for Infection Prevention and Control, Samsung Medical Center, Seoul, ${ }^{5}$ Division of Infectious Diseases, Department of Internal Medicine, Samsung Medical Center, Sungkyunkwan University School of Medicine, Seoul, Korea

Purpose: Tuberculosis (TB) is one of the most important diseases that cause significant mortality and morbidity in young children. Data on TB transmission from an infected child are limited. Herein, we reporta case of disseminated TB in a child and conducted a contact investigation among exposed individuals. Methods: A 4-year-old child without Bacille Calmette-Guérin vaccination was diagnosed as having culture-proven disseminated TB. The child initially presented with symptoms of inflammatory bowel disease, and nosocomial and kindergarten exposures were reported. The exposed individuals to the index case were divided into 3 groups, namely household, nosocomial, or kindergarten contacts. Evaluation was performed following the Korean guidelines for TB. Kindergarten contacts were further divided into close or casual contacts. Chest radiography and tuberculin skin test or interferon-gamma-releasing assay were performed for the contacts.

Results: We examined 327 individuals (3 household, 10 nosocomial, and 314 kindergarten contacts), of whom 18 (5.5\%), the brother of the index patient, and 17 kindergarten children were diagnosed as having latent TB infection (LTBI). LTBI diagnosis was more frequent in the children who had close kindergarten contact with the index case (17.1\% vs. 4.4\%, $P=0.007)$. None of the cases had active TB.

Conclusion: This is the first reported case of TB transmission among young children from a pediatric patient with disseminated TB in Korea. TB should be emphasized as a possible cause of chronic diarrhea and failure to thrive in children. A national TB control policy has been actively applied to identify Korean children with LTBI.

Key words: Disseminated tuberculosis, Inflammatory bowel disease, Contact investigation

\section{Introduction}

Tuberculosis (TB) is still one of the most important diseases causing significant mortality and morbidity in young children. In 2015, the estimated number of deaths due to TB in children younger than 5 years was 191,000 worldwide. ${ }^{1)}$

In Korea, the incidence rate of TB in 2017 was 0.7 patients per 100,000 population aged $<5$ years, which had decreased by 59\% from 2013. ${ }^{2)}$ Bacille Calmette-Guérin (BCG) vaccination is recommended for Korean children as a part of a national immunization program (NIP) and 2 strains (Danish-1331, Tokyo-172) are currently used. BCG vaccination in the neonatal period has been shown to prevent $82 \%$ of pulmonary TB, TB meningitis, and disseminated TB in
Corresponding author: Yae-Jean Kim, MD, PhD Department of Pediatrics, Samsung Medical Center, Sungkyunkwan University School of Medicine, 81 Irwon-ro, Gangnam-gu, Seoul 06351, Korea Tel: +82-2-3410-0987

Fax: +82-2-3410-0043

E-mail: yaejeankim@skku.edu https://orcid.org/0000-0002-8367-3424

Received: 25 October 2018

Revised: 9 November 2018

Accepted: 13 November 2018

This is an open-access article distributed under the terms of the Creative Commons Attribution NonCommercial License (http://creativecommons.org/ licenses/by-nc/4.0/) which permits unrestricted noncommercial use, distribution, and reproduction in any medium, provided the original work is properly cited. 
children under 15 years old. ${ }^{3)}$

Disseminated TB is a severe type of TB defined as isolation of Mycobacterium tuberculosis from blood or bone marrow, from a liver biopsy specimen, or from specimens in 2 noncontiguous organs in a single patient. ${ }^{4)}$ The incidence and infectivity of disseminated TB in immunocompetent pediatric patients is poorly understood.

We experienced an immunocompetent, BCG unvaccinated child who presented with symptoms mimicking clinical manifestations of inflammatory bowel disease and was later diagnosed with disseminated TB. Thorough contact investigations were performed among household, nosocomial, and community exposures.

\section{Materials and methods}

\section{Index case}

A 4-year-old girl visited the pediatric gastroenterology outpatient clinic with recurrent abdominal pain and chronic diarrhea for the past year. She had previously been healthy until she developed these symptoms. Her height was $104 \mathrm{~cm}$ (25th-50th percentile) and weight was $13 \mathrm{~kg}$ (less than 3rd percentile). She did not have any fever or cough. An inflammatory bowel disease and resulting malnutrition were highly suspected as an initial diagnosis. Her white blood cell count was 7,820/ $\mathrm{L}$ (neutrophil 59\%, lymphocyte 39\%), hemoglobin (Hb) level was $7.8 \mathrm{~g} / \mathrm{dL}$, and platelet count was 571,000/ $\mu \mathrm{L}$. Her C-reactive protein level was $5.9 \mathrm{mg} / \mathrm{dL}$ (normal range, 0$0.3 \mathrm{mg} / \mathrm{dL}$ ). Stool occult blood was positive and calprotectin in stool specimen was markedly elevated ( $>1,000 \mu \mathrm{g} / \mathrm{g}$; normal range, $<50$ $\mu \mathrm{g} / \mathrm{g}$ ). Abdominal computed tomography (CT) performed in the evening of the day of admission revealed multiple necrotic lymphadenopathy with peritonitis and wall thickening of colon (Fig. 1A, B). Additional chest CT was performed emergently and the patient was put into isolation. Chest CT showed multiple calcified mediastinal, hilar lymphadenopathy, and tree-in-bud opacity in both lungs (Fig. 1C, D).

After the chest CT, the parents revealed the additional history information that she had not received any vaccination including BCG after birth and had been treated only with herbal medication by a traditional healer for her condition. Of note, a close relative (grandaunt) was diagnosed with pulmonary TB when the child was 15 months old.

Based on this history and radiologic findings, TB was strongly suspected. Acid fast bacilli (AFB) staining with culture was performed for 3 consecutive days with gastric aspirates. No AFB were observed by staining and a polymerase chain reaction test was also negative. Colonoscopy showed skipped lesions of ulcers and pseudopolyps from proximal transverse to descending colon (Fig. 2). Pathologic findings of the biopsy specimens from colon showed active inflamed granulation tissues. Cerebrospinal fluid study was normal and bone scan did not show any abnormal uptake. Her interferon gamma releasing assay (IGRA) test was positive.

After initial evaluations, she immediately started anti-TB medication. After 5 days of treatment the abdominal pain improved and her oral intake increased. She was discharged after 7 days of anti-TB treatment and continued isolation at home. Of note, she

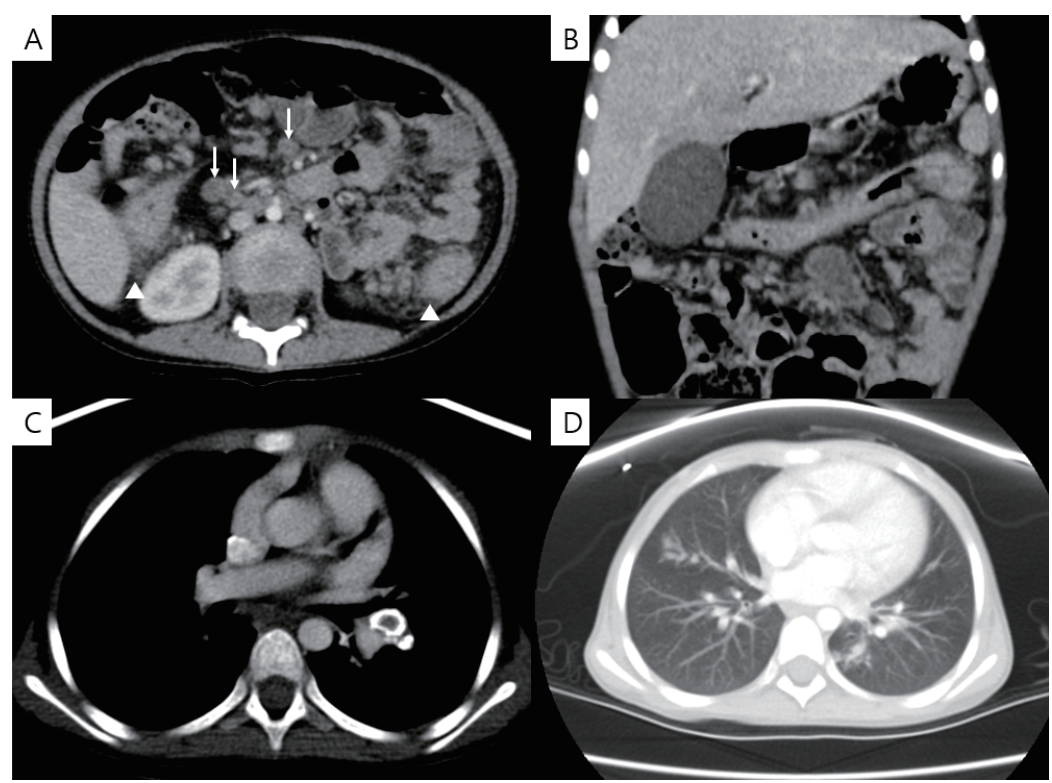

Fig. 1. Abdominal and chest computed tomography scans. (A) Multiple mesenteric lymphadenopathy with necrosis (white arrow), pericolic fat infiltration (white triangle), (B) wall thickening of the transverse colon, $(\mathrm{C})$ mediastinal egg-shell shaped calcified lymph node, and (D) tree-in-bud appearance. 
was apathetic and did not show any emotional response for communication on the admission day. However, she showed emotional responses with facial expressions including smiling at discharge. Her body weight at discharge was $14.8 \mathrm{~kg}$. Cultures from all 3 gastric juice specimens finally grew $M$. tuberculosis after 17 days and 2 out of 5 colon biopsy specimens grew $M$. tuberculosis 16 days later. Isolates were sensitive to all anti-TB medicines.

A whole body magnetic resonance imaging (MRI) scan was performed to investigate other organ involvement. Although a previous bone scan showed negative findings, MRI showed diffuse abnormal bone marrow signal changes on axial and appendicular skeleton highly suggestive of bone marrow involvement of TB.

After 8 months of treatment, the patient showed marked improvement of anemia and nutritional status. Her height was $110.8 \mathrm{~cm}$ (50th-75th percentile), weight was $17.6 \mathrm{~kg}$ (25th-50th percentile), and $\mathrm{Hb}$ level was $13.3 \mathrm{~g} / \mathrm{dL}$. She started childhood vaccination with an accelerated schedule according to Korean NIP.

\section{Contact investigation}

Exposed individuals were divided into 3 groups of household, nosocomial, and kindergarten contacts. Investigations were performed according to the Korean guidelines for TB. ${ }^{5)}$ Data on kindergarten contacts was provided by Korean Center for Disease Control and Prevention (KCDC).

All exposed individuals underwent chest X-ray and tuberculin skin tests (TSTs) or IGRA tests were also performed. Contacts with known positive results for TST or IGRA were tested only with chest

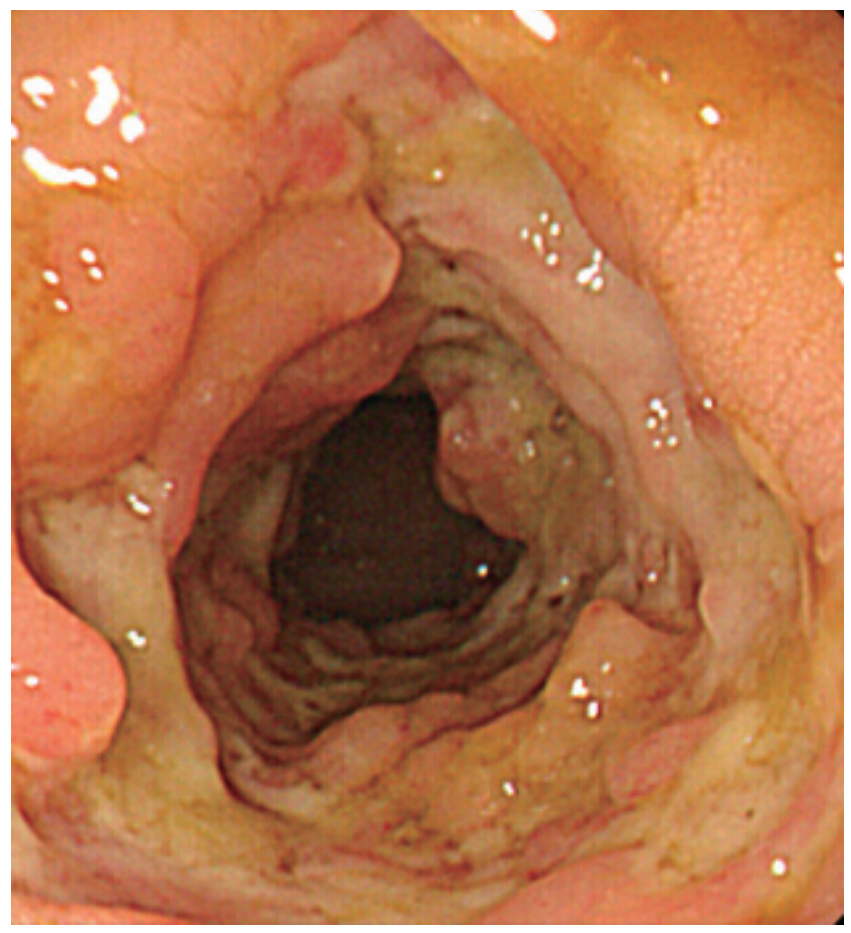

Fig. 2. Ulcers and pseudopolyps of the colon.
X-ray. (for example, health care workers who had a positive result from regular TB screening).

Household and nosocomial contacts were considered close contacts. Children younger than 5 years received a serial TST (2 TST with 8 - to 10 -week interval). Adults and children aged $\geq 5$ underwent testing with TB IGRA.

Kindergarten contacts were divided into 2 groups of close and casual contacts. Among children, close contact was defined as sharing the same classroom for more than 8 hours a day or 40 hours in total. Children in the same class or adjacent class sharing the aisle, and those riding the same bus were included in this group. Casual contacts were defined as other children in the same kindergarten. Among adults, teachers of the class of the index patient and the bus driver were classified as close contacts. Casual contacts were defined as all other adult employees. Children were tested with TST and adults were evaluated with IRGA. Pediatric close contacts were evaluated with a serial TST. Pediatric casual contacts were evaluated with one TST after 8 weeks of last exposure. Adult casual or close contacts without previous positive IGRA test results were evaluated with one IGRA test after 8 weeks of last exposure. The Institutional Review Board at Samsung Medical Center, Seoul, South Korea reviewed and approved this study (approval number: 201809-028-002). The chi-square test was used to analyze categorical variables. A $P$ value of $<0.05$ was considered statistically significant. All statistical analysis was performed using the $\mathrm{R}$ ver. 3.4.3 (R Foundation for Statistical Computing, Vienna, Austria).

\section{Results}

A flow chart for contact investigation is shown in Fig. 3.

Among all exposed individuals, 18 individuals (5.5\%) were identified as LTBI patients. There were no cases of active TB.

\section{Household exposure}

The patient's parents did not have any evidence of TB infection, with normal chest X-ray and negative IGRA results. Her 12-monthold brother also did not receive the BCG vaccine and was thoroughly evaluated. He was diagnosed with latent TB and was placed on isoniazid treatment for 9 months. Her brother also received childhood vaccination of accelerated schedule according to Korean NIP.

\section{Nosocomial exposure}

The patient was initially admitted to a shared room with other children on a general ward but was isolated after 19 hours when TB was suspected. Because of this nosocomial exposure, a contact investigation was performed among other pediatric patients $(n=4)$ and adults $(n=6)$ who were considered to have significant exposure. These individuals were followed up for 2 months. Chest X-ray and serial TB-specific IGRA tests were performed at 8- to 10-week in- 


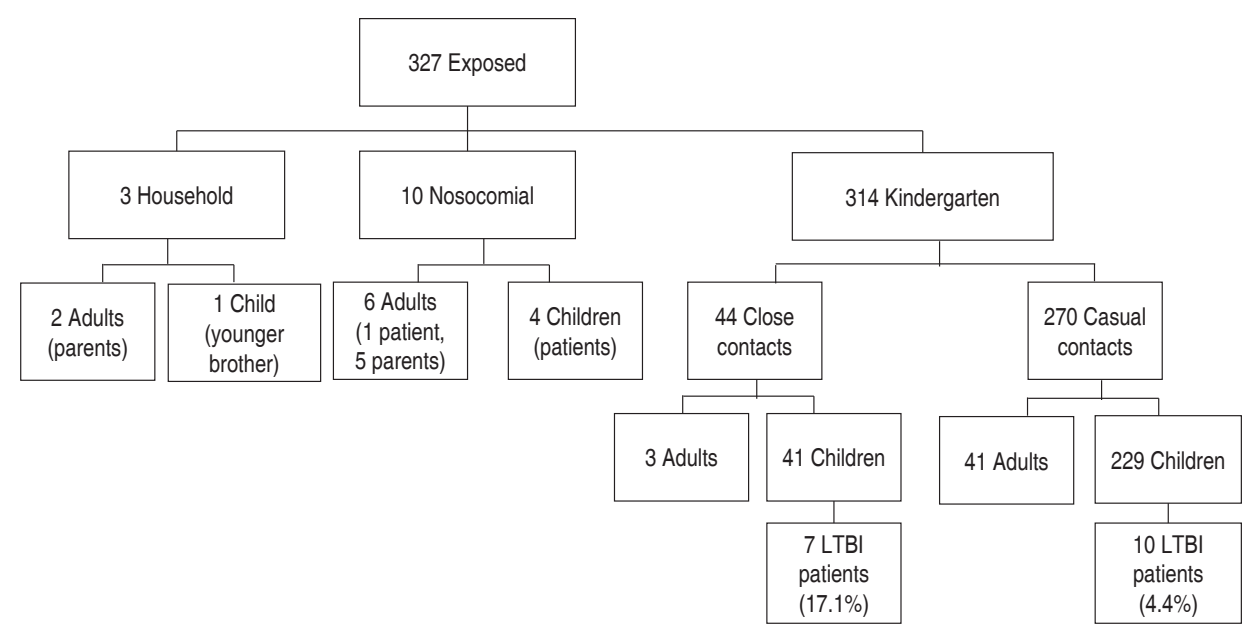

Fig. 3. Exposure investigation flow. LTBI, latent tuberculosis infection.

tervals. At 3 months, none of these individuals was identified as infected with TB from this exposure. Her TB infection was reported to the health authority and contact investigations in her family and kindergarten were also performed by the KCDC.

\section{Community exposure}

A total of 314 exposed individuals were investigated: 44 adults and 270 children. Data on demographic information, extent of exposure, and assessment results of kindergarten children are shown in Table 1.

Of all exposed children, 257 (95.1\%) completed LTBI tests and 17 (6.3\%) of all exposed were diagnosed with LTBI. LTBI diagnosis was more frequent in children who had close contact with the index case (7 of 41 [17.1\%] close contacts compared with 10 of 229 [4.4\%] casual contacts; $P=0.007$ ).

Among the 229 casual contacts of all ages, LTBI was diagnosed in $4.8 \%$ ( 2 of 42) of children younger than 5 years and 4.3\% (8 of 187) of children 5 years or older $(P=0.9)$.

There were no LTBI cases from this exposure among adults. As of 12 months from the investigation, there was no active TB case from this exposure.

\section{Discussion}

In this study, after diagnosis of a single case of disseminated TB infection, an extensive contact investigation was performed for household, nosocomial, and community exposures. Our index patient was a 4-year-old girl who had symptoms mimicking inflammatory bowel disease. Even though she did not have significant cough or cavitary lung lesions at the time of TB diagnosis, the investigation revealed TB transmission among $6.3 \%$ of exposed children at her kindergarten.

There are limited data in the literature on TB transmission among
Table 1. Characteristics of children with kindergarten contact with the index case

\begin{tabular}{lccc}
\hline Characteristic & Number & No. completing LTBI ${ }^{*}$ test & LTBI, n (\%) \\
\hline Total & 270 & 257 & $17(6.3)$ \\
Male & 152 & & $8(5.3)$ \\
Female & 118 & $9(7.6)$ \\
Age (yr) & & \\
3 & 6 & $2(33.3)$ \\
4 & 77 & $7(9.1)$ \\
5 & 101 & & $5(5.0)$ \\
$\geq 6$ & 86 & 32 & $3(3.5)$ \\
Extent of exposure $^{\dagger}$ & & & $7(17.1)$ \\
Close $^{\dagger}$ & 41 & 225 & $10(4.4)$ \\
Casual $^{\ddagger}$ & 229 & & \\
\hline
\end{tabular}

LTBI, latent tuberculosis infection - positive tuberculin skin test $(\geq 10 \mathrm{~mm}$ of induration or increased diameter of induration by $>6 \mathrm{~mm}$ at second test) or interferon gamma releasing assay results with normal chest radiograph without symptoms of active tuberculosis. ${ }^{\dagger}$ Close contacts: children in the same class or adjacent class sharing the aisle, and those riding the same bus. ${ }^{\ddagger}$ Casual contacts: other children in the kindergarten.

children from a pediatric TB index case less than 5 years of age, ${ }^{6,7)}$ although there have been several studies investigating exposure among adolescents. ${ }^{8,9}$ In our investigation, the index case and many of the contacts were children aged less than 5 years with a high risk for severe TB diseases. Young children are considered to have lower infectivity than adults because they do not generate a forceful cough to be infectious. ${ }^{10)}$ Known risk factors for TB transmission are severe cough, cavitary lung lesions, closer and longer contacts, and delayed diagnosis of $\mathrm{TB}^{8,11)}$ Although the index child in our study did not have severe cough or cavitary lung lesions, she had prolonged contact with other young children before the diagnosis of her disseminated TB.

In Korea, since 2013 there has been a nationwide program to strengthen TB contact investigation and a TB control policy as part 
of efforts to decrease the TB disease burden in the country. A possible source of transmission to this child was a close relative who was diagnosed with active pulmonary TB when the child was 15 months old. This study suggests that a proactive TB contact investigation should be performed in exposed contacts with high risk for serious TB progression (e.g., young children).

Taking a history of routine vaccination status and illness in the family is always important for all pediatric patients. Of note, this child had antivaccine parents and did not receive BCG or any other vaccination included in the Korean NIP. The parents' refusal of the vaccine and reluctance to appropriate medical treatment resulted in life-threatening TB infection in the child herself and a public health threat to many other young children in the community. Although there was no active TB case, $6.3 \%$ of all exposed children and 17.1\% of children with close contact in her kindergarten developed LTBI.

It is of note that IBD was initially suspected in this child since she had prolonged diarrhea, poor weight gain and high level of calprotectin. The incidence of TB among pediatric patients in Korea is decreasing; the incidence rate of TB among Korean children younger than 5 years was 0.7 patients per 100,000 population in 2017. However, the incidence rate of IBD among children has been increasing in Korea and many other parts of the world. ${ }^{12,13)}$ Because of increased awareness of early onset pediatric IBD, many clinicians now suspect IBD more often than TB in pediatric patients with chronic diarrhea and stunted growth. There is a similar trend in adult patients. A study of Korean adults showed that the number of cases of intestinal TB misdiagnosed as Crohn disease (CD) has increased and the number of cases of $\mathrm{CD}$ misdiagnosed as intestinal TB has decreased. ${ }^{14)}$ Delayed diagnosis and treatment of TB increases morbidity and mortality. ${ }^{15)}$ Although the incidence of TB is decreasing, the overall incidence rate of TB in individuals of all ages in Korea was still 55.0 per 100,000 in 2017. ${ }^{2)}$ Therefore, children in Korea are at higher risk of TB exposure compared to those in countries with a low TB incidence rate of fewer than 10 per 100,000 population.

Limitations of this study include the lack of follow-up records and BCG vaccination status. Some patients with LTBI did not start treatment. However, there was no reported case of active TB during 12 months after the investigation.

This is the first report of TB transmission among young children from a pediatric patient with disseminated TB in Korea. TB should be re-emphasized as a possible cause for chronic diarrhea and failure to thrive in children. The national TB control policy has been actively applied to identify LTBI in Korean young children.

\section{Conflict of interest}

No potential conflict of interest relevant to this article was reported.

\section{References}

1. Dodd PJ, Yuen CM, Sismanidis C, Seddon JA, Jenkins HE. The global burden of tuberculosis mortality in children: a mathematical modelling study. Lancet Glob Health 2017;5:e898-906.

2. Park WS, Cha JO, Jang S, Kim J, Cho KS. Tuberculosis notification status in Korea in 2017. Public Health Wkly Rep 2018;11:401-6.

3. Comstock GW. Does the protective effect of neonatal BCG vaccination correlate with vaccine-induced tuberculin reactions? Am J Respir Crit Care Med 1996;154:263-4.

4. Crump JA, Reller LB. Two decades of disseminated tuberculosis at a university medical center: the expanding role of mycobacterial blood culture. Clin Infect Dis 2003;37:1037-43.

5. Joint Committee for the Revision of Korean Guidelines for Tuberculosis; Korea Centers for Disease Control and Prevention. Korean guidelines for tuberculosis. 3rd ed. Seoul (Korea): Joint Committee for the Revision of Korean Guidelines for Tuberculosis, Korea Centers for Disease Control and Prevention, 2017.

6. Lee EH, Graham PL 3rd, O'Keefe M, Fuentes L, Saiman L. Nosocomial transmission of Mycobacterium tuberculosis in a children's hospital. Int J Tuberc Lung Dis 2005;9:689-92.

7. Muneef MA, Memish Z, Mahmoud SA, Sadoon SA, Bannatyne R, Khan Y. Tuberculosis in the belly: a review of forty-six cases involving the gastrointestinal tract and peritoneum. Scand J Gastroenterol 2001;36:528-32.

8. Phillips L, Carlile J, Smith D. Epidemiology of a tuberculosis outbreak in a rural Missouri high school. Pediatrics 2004;113:e514-9.

9. Anaraki S, Bell AJ, Perkins S, Murphy S, Dart S, Anderson C. Expected background rates of latent TB infection in London inner city schools: lessons from a TB contact investigation exercise in a secondary school. Epidemiol Infect 2018;146:2102-6.

10. Starke JR. Transmission of Mycobacterium tuberculosis to and from children and adolescents. Semin Pediatr Infect Dis 2001;12:115-23.

11. Kaplan G, Post FA, Moreira AL, Wainwright H, Kreiswirth BN, Tanverdi M, et al. Mycobacterium tuberculosis growth at the cavity surface: a microenvironment with failed immunity. Infect Immun 2003;71:7099-108.

12. Hong SJ, Cho SM, Choe BH, Jang HJ, Choi KH, Kang B, et al. Characteristics and incidence trends for pediatric inflammatory bowel disease in Daegu-Kyungpook Province in Korea: a multi-center study. J Korean Med Sci 2018;33:e132.

13. Benchimol EI, Fortinsky KJ, Gozdyra P, Van den Heuvel M, Van Limbergen J, Griffiths AM. Epidemiology of pediatric inflammatory bowel disease: a systematic review of international trends. Inflamm Bowel Dis 2011;17:423-39.

14. Seo H, Lee S, So H, Kim D, Kim SO, Soh JS, et al. Temporal trends in the misdiagnosis rates between Crohn's disease and intestinal tuberculosis. World J Gastroenterol 2017;23:6306-14.

15. Ahmad M, Ahmed A. Tuberculous peritonitis: fatality associated with delayed diagnosis. South Med J 1999;92:406-8. 